\title{
Impacto del grado de satisfacción en las expectativas del estudiante universitario. El caso de la Universidad Autónoma de Chiapas (UNACH)
}

\section{Impact of the degree of satisfaction on the expectations of the university student. The case of the Autonomous University of Chiapas (UNACH)}

\author{
OVANDO-DÍAZ, Marco Antonio $\dagger$, GRAJALES-CASTILLEJOS, Octavio y MENA-ÁLVAREZ, \\ Andrea
}

Universidad Autónoma de Chiapas, Facultad de Humanidades

ID $1^{\text {er }}$ Autor: Marco Antonio, Ovando Díaz, CVU CONACYT ID: 994381

ID $1^{\mathrm{er}}$ Coautor: Octavio, Grajales Castillejos / CVU CONACYT ID: 289361

ID $2^{\text {do }}$ Coautor: Andrea, Mena Álvarez / CVU CONACYT ID: 643343

DOI: $10.35429 /$ JPDL.2019.16.5.20.27

Recibido 10 de Junio, 2019; Aceptado 30 Septiembre, 2019

Resumen

El objetivo general del artículo es determinar el grado de satisfacción de los estudiantes de la Universidad Autónoma de Chiapas, México, a través de un estudio de corte transversal para valorar los servicios institucionales que ofrece y su impacto en su formación académica. Los objetivos específicos son: 1) Analizar la opinión de los alumnos respecto a su plan de estudios y profesores, 2) Analizar la opinión de los alumnos respecto al conocimiento de la normatividad universitaria y 3) Describir las condiciones de infraestructura Universitaria: física y tecnológica. La metodología utilizada fue el método explicativo de corte cualitativo-cuantitativo y trasversal, para ello fue necesario el diseño y la aplicación de una encuesta tipo Likert, la cual se aplicó a 3 mil 842 estudiantes inscritos del 5to. al 8 vo. semestre de 22 Licenciaturas de las diversas sedes de la UNACH. La contribución que esta investigación tiene es: a) Brindar elementos académicos a las autoridades universitarias para la toma de decisiones en sus procesos de acreditación, b) Formación de recursos humanos a través de la participación de los alumnos en el desarrollo de la investigación y c) Sensibilizar a la comunidad universitaria a través de la publicación de los resultados de la investigación.

Calidad de la educación, Estudiante Universitario, Acreditación

\begin{abstract}
The general objective of the article is to determine the degree of satisfaction of the students of the Autonomous University of Chiapas, Mexico, through a cross-sectional study to assess the institutional services offered and their impact on their academic training. The specific objectives are: 1) Analyze the opinion of students regarding their curriculum and teachers, 2) Analyze the opinion of students regarding the knowledge of university regulations and 3) Describe the conditions of University infrastructure: physical and technological. The methodology used was the explanatory method of qualitative-quantitative and transverse cutting, for this it was necessary to design and apply a Likert-type survey, which was applied to 3,842 enrolled students of the 5th. to the 8 th. semester of 22 Bachelor's degrees from the various UNACH headquarters. The contribution that this research has is: a) Provide academic elements to university authorities for decision making in their accreditation processes, b) Training of human resources through the participation of students in the development of research and c) Sensitize the university community through the publication of research results.
\end{abstract}

Quality of education, University Student, Accreditation.

Citación: OVANDO-DÍAZ, Marco Antonio, GRAJALES-CASTILLEJOS, Octavio y MENA-ÁLVAREZ, Andrea. Impacto del grado de satisfacción en las expectativas del estudiante universitario. El caso de la Universidad Autónoma de Chiapas (UNACH). Revista de Filosofía y Cotidianidad. 2019, 5-16: 20-27

\footnotetext{
* Correspondencia del Autor (marcoovando@hotmail.com)

$\uparrow$ Investigador contribuyendo como primer autor.
} 


\section{Introducción}

El presente artículo, da cuenta en términos generales de los resultados obtenidos en relación al estudio realizado: "Impacto del grado de satisfacción en las expectativas del estudiante universitario. El caso de la Universidad Autónoma de Chiapas", el cual se encuentra en la etapa de sistematización y análisis de la información.

El propósito general del proyecto pretendía comprobar hasta donde los efectos del conocimiento recibido y las condiciones de los servicios institucionales han contribuido a satisfacer las necesidades y expectativas del estudiante universitario. Los resultados se presentan mediante las categorías siguientes: Plan de estudio y profesor, Normatividad e Infraestructura física.

Aunque no se aportan nuevos elementos metodológicos en el estudio, es importante aclarar que la intención es brindar información confiable a las autoridades universitarias para la toma de decisiones, teniendo como base técnicas e instrumentos ya probados en investigaciones realizadas; cabe destacar que en la encuesta las preguntas se formularon acorde al contexto de ésta universidad.

Desde el momento de la elaboración del protocolo, se pensó en diseñar la metodología, las técnicas y la encuesta para que a partir de los datos obtenidos se evaluará como hipótesis cómo el nivel de satisfacción de los estudiantes universitarios está condicionada por los contenidos curriculares, el profesor, la infraestructura y son detonantes para determinar sus expectativas profesionales como egresados.

El propósito no fue solucionar el problema, más bien desde un principio se tuvo clara la idea de aportar información desde la opinión de los estudiantes acerca de las expectativas del universitario y conformar una base de datos con su respectivo análisis para que las autoridades pudieran proporcionar información a los organismos evaluadores que existen en nuestro país.

\section{El imaginario social, la representación social y su nexo con el plan de estudios y profesor}

Se parte de la premisa de que se puede afirmar que los jóvenes actúan de manera paralela en su entorno social donde se rodea de sus actividades cotidianas que enfrenta para actuar de acuerdo a sus intereses y expectativas alcanzando logros en su vida y generar un cambio de racionalidad y transformación en su vida profesional y laboral, conformando una identidad que va creando en su concepción de cómo percibe el mundo que lo rodea y un equilibrio de su personalidad hacia los demás por medio de la socialización en su entorno.

Baeza (2000) afirma:

Los imaginarios sociales son composiciones ya socializadas en el tramado mismo de las relaciones sociales, con el propósito de dar inteligibilidad al cosmos, al mundo y a la sociedad, al mundo y a la naturaleza, a la vida desde sus orígenes y a la muerte, etc. El hombre consubsustancialmente combate su propia perplejidad. (p 33)

Carretero (2001) aporta elementos en relación a la conceptualización del imaginario social cuando incorpora el concepto de autonomía y explica:

En la perspectiva en la que nos movemos, interesa destacar que lo imaginario recupera una autonomía a partir de lo cual no se resigna a ser aprehendido desde ninguna actitud reduccionista, que lo convierta en la expresión de una carencia real y un sustituto irreal a dicha carencia. Desde este punto de vista, la esencia de lo imaginario adquiere un carácter propio, no se concibe como una consecuencia derivada de una causa siempre real, tal como es analizado en otros discursos teóricos contemporáneos, sino como un orden experiencial diferente y con una lógica propia. (págs. 123 y124).

Con base en lo anterior, el imaginario social es una capacidad imaginante, o bien, una producción de significaciones colectivas, que al ser procedente se va transformando en distintas categorías de representaciones imaginarias como característica propia de la vida en sociedad. 
No obstante, los autores señalados indican que el imaginario social, se presenta como una representación de las ideas que el sujeto personifica en su cotidianidad, a lo largo de los procesos históricos que va transformando en el momento que cambia a su realidad. Por lo tanto, la imaginación social es construida e interpretada por cada sujeto en un momento histórico de su vida social.

En este sentido de otorgarle connotación al mundo, se puede marcar como ejemplo de abstracción lo relacionado con el plan de estudios y los profesores ya que el imaginario permite crear el escenario o expectativas a partir de significaciones para comprender y dar forma a la idea que se tiene del plan de estudios y profesores, contando con la experiencia o de la convivencia que se tiene de ellos, lo que permite seguir construyendo ideas más claras de manera socio - cognitiva.

En el caso de las representaciones sociales han sido desarrolladas a partir de las concepciones de Moscovici (1979). Dicho autor incluso advertía la complejidad existente para una definición precisa por poseer una "posición mixta" al ser una especie de "encrucijada" de conceptos sociológicos y psicológicos. Sin embargo, desarrolla toda una teoría desde la psicología social en el que argumenta.

La representación social es una modalidad particular del conocimiento, cuya función es la elaboración de los comportamientos y la comunicación entre los individuos. La representación es un corpus organizado de conocimientos y una de las actividades psíquicas gracias a las cuales los hombres hacen inteligible la realidad física y social, se integran en un grupo o en una relación cotidiana de intercambios, liberan los poderes de su imaginación" (Moscovici,1979 p.17).

En este orden de ideas el aporte que hace Vergara (2008) clarifica la idea de las representaciones sociales.

Las representaciones sociales se relacionan con el convencimiento de que son guía de acción y marco de lectura de la realidad. Son signifcaciones que permiten interpretar el curso de los acontecimientos y las actuaciones sociales.
Expresan las relaciones que las personas mantienen con el mundo y con los otros. Relaciones que son forjadas en la interacción y el contacto con los discursos que circulan en el espacio público. Son programas de percepción, construcciones con estatus de teoría. Y en este sentido no son un saber apodíctico, pero contienen un saber válido. Es por esto que la aproximación a las representaciones sociales se constituye en un aparato teórico heurístico para profundizar en el conocimiento de la realidad social. (pág. 62).

Por su parte Jodelet (1986) afirma:

Las representaciones sociales constituyen modalidades de pensamiento práctico orientadas hacia la comunicación, la comprensión y el dominio del entorno social, material e ideal. En tanto que tales, presentan características específicas a nivel de organización de los contenidos, las operaciones mentales y la lógica" (pág. 474).

Por su parte, se puede decir que las representaciones sociales, son decodificaciones porque reúnen un conjunto de anticipaciones y expectativas, es decir, el individuo debe tener una experiencia previa que le fijará esa representación, he ahí lo subjetivo; lo colectivo será porque esa representación dependerá de la sociedad en la cual vive, pues eso mediará la forma de vivirla, actuarla y representarla, es una dualidad subjetiva y social.

Parafraseando los términos en los que los autores señalados plantean el tema de la satisfacción del estudiante universitario en su formación académica, podemos inferir que los jóvenes actúan en función a su entorno social que los rodea en sus actividades cotidianas que se enfrentan y de esa manera actúan de acuerdo a sus intereses y expectativas que pretende lograr en su vida para generar un cambio de racionalidad y transformación en su vida profesional y laboral, por una identidad que va creando en su concepción de cómo percibe el mundo que lo rodea y un equilibrio de su personalidad hacia los demás por medio de la socialización en su entorno. 
De manera particular, la aplicación del imaginario social y las representaciones sociales se pueden advertir en la construcción y desarrollo del plan de estudio en donde se establecen las competencias que se quieren formar en el estudiante y las expectativas que la institución se crea al establecer sus objetivos y contenidos a implementar; en este sentido, los actores (autoridades, profesores y alumnos) planean, organizan y especifican las tareas a desarrollar y las prácticas educativas. Claro está que el profesor juega un papel importante en tanto tratará de cumplir con las expectativas establecidas curricularmente y las aspiraciones de los estudiantes.

\section{Las expectativas del estudiante y su marco normativo}

En este orden de ideas, se puede advertir que en el contexto institucional de la vida universitaria las expectativas del universitario se tienen que enmarcar a las normas establecidas por la dependencia en donde estudia, a los profesores y a sus compañeros con quienes estudia.

Se puede deducir que el imaginario social de los estudiantes universitarios dentro de la institución en cuanto a su formación académica se presenta a través de las estructuras mentales que tiene el sujeto en su personalidad planteando aspectos trascendentes a lo largo de su carrera. Las representaciones sociales sobre el saber, el conocimiento, la formación, la enseñanza y el aprendizaje, el examen y atribuciones que realizan los estudiantes acerca de los docentes, permite establecer un sistema de pre codificación de la realidad que orienta las actitudes, comportamientos y prácticas, y permite a los sujetos una justificación de toma de posición y comportamiento adoptados en la institución.

Las atribuciones dirigidas hacia los otros, hacia el propio grupo, cumplen un papel similar respecto a las actitudes, comportamientos y prácticas: inducen un sistema de anticipaciones y de expectativas, seleccionando informaciones e interpretaciones que van construyendo una realidad coherente. Son asimismo, prescriptivas de comportamientos en tanto definen lo que es lícito, tolerable, adecuado, aceptable o inaceptable en un contexto cultural e institucional determinado.
No se trata, entonces, acerca del "reflejo" de una realidad externa al sujeto, sino de una organización significante que, aunque no es ajena a factores contingentes propios de la situación y el contexto, y a las constricciones que ellos imponen, supone una elaboración e interpretación. Las instituciones instauran mecanismos que tienen efectos en la producción de subjetividad. Esos dispositivos están constituidos por normas, valores, prácticas, rituales y discursos que traducen relaciones de poder los cuales regulan actitudes así como comportamientos. Esos valores, normas, prácticas y discursos de los sujetos se apoyan en sistemas de significación de carácter colectivo, propios de una cultura. En cuanto a la satisfacción y expectativas del estudiante universitario conceptualmente se puede considerar lo que señala Surdez, et al (2018 p. 12 y 13): "La satisfacción estudiantil se puede conceptualizar como el bienestar que experimentan los alumnos por sentir cubiertas sus expectativas académicas como resultado de las actividades que realiza la institución para atender sus necesidades educativas". Lo grave sería no tomar decisiones que contribuyan a su mejoramiento de acuerdo con los datos encontrados en investigaciones realizadas; en este sentido, sería equivalente a no tenerla en cuenta. Por su parte, Alves y Raposo (2005), indican que:

La satisfacción del alumno en los estudios universitarios ha cobrado vital importancia para las instituciones de este sector, pues de ella depende su supervivencia. Solo con la satisfacción de los alumnos se podrá alcanzar el éxito escolar, la permanencia de los estudiantes en la institución, y sobre todo, la formación de una valoración positiva boca a boca. En este sentido, es extremamente importante encontrar formas fiables de medir la satisfacción del alumno en la enseñanza universitaria, permitiendo así a las instituciones de enseñanza conocer su realidad, compararla con la de los otros competidores y analizarla a lo largo del tiempo ( $p$ 74).

Por lo tanto, no se puede descartar el hecho de que la satisfacción del estudiante con la formación que reciben es constantemente referida como un elemento clave en la valoración de la calidad de la educación ya que son los estudiantes quienes sirven como referente fundamental a la hora de establecer los estándares de calidad del servicio que se ofrece. 
En este sentido, es importante que las universidades realicen estudios sobre la satisfacción de sus estudiantes pues permiten analizar la calidad de estas instituciones e inferir características importantes del funcionamiento de los planes de estudios que imparten.

\section{La satisfacción del estudiante y su relación con la infraestructura física}

Se presupone que la satisfacción del estudiante universitario pone en evidencias hasta donde están complacidos de los servicios académicos, de infraestructura física y tecnológica, de los servicios de extensión, vinculación e estudiantiles, entre otros. En este sentido, se puede contar con información que permita evaluar la pertinencia de las funciones sustantivas y adjetivas que oferta la universidad valorando el grado de eficiencia así como eficacia y su impacto en la formación de los universitarios y su relevancia con la sociedad.

En esta lógica es evidente que no es la opinión de los estudiantes el único medio para evaluar a una institución, pero debe tenerse en cuenta también, puesto que ellos son el objeto de la institución y no sólo en relación a determinados elementos o aspectos de la vida universitaria, sino a todos aquellos que incidan directa o indirectamente en su calidad educativa. Se puede inferir que los estudios de satisfacción de los estudiantes universitarios son importantes porque también pueden proporcionar datos e incidir en la toma de decisiones para la disminución de las tasas de fracaso y abandono escolar, entre otros aspectos.

Por último se cita a Jiménez, Terriquez y Robles (2011) quienes indican:

Los estudiantes son los principales usuarios de los servicios universitarios y los destinatarios de la educación, por lo tanto son ellos los que mejor pueden valorarla y, aunque pueden tener una visión parcial, sus opiniones no dejan de ser fruto de sus percepciones, influenciadas por expectativas, necesidades y por diversos factores, que sirven como indicador para la mejora de la gestión y el desarrollo de los programas académicos. (pág 46).
Se plantea que el debate teórico y metodológico sigue abierto sobre todo que da la oportunidad de seguir construyendo metodologías que permitan aplicarse para continuar escudriñando la forma de pensar y actuar del estudiante universitario y como las instituciones educativas tienen el reto de atender sus necesidades y expectativas de formación.

\section{Metodología a desarrollar}

Uno de los propósitos del estudio es analizar y predecir los datos recabados a la comunidad universitaria de la UNACH, se utiliza la metodología de corte cuantitativo, en el cual se diseñó e implementó una encuesta a los alumnos del 5to. Al 8vo. semestre de 22 Licenciaturas, la cual permitió identificar las espectativas del estudiante universitario. Una vez diseñado el instrumento de recolección de la información, se aplicaron a 3 mil 842 estudiantes, de donde se recabó información cuantitativa, haciendo uso del programa SPSS, en donde se capturaron e interpretaron los datos.

\section{Resultados}

En este apartado se analiza la información recabada de los estudiantes universitarios quienes fueron encuestados para conocer su opinión respecto a los servicios que la Universidad les ha ofrecido durante su formación académica. Los resultados aquí vertidos, se avocan a tres categorías: Plan de estudios y profesor, Normatividad e Infraestructura física.

\section{Plan de estudios y profesor}

Plan de estudios

Los porcentajes obtenidos muestran que la mayoría de los alumnos $(60.5 \%)$ conocen bien el Plan de Estudios de su carrera respectiva, el $20.7 \%$ lo conoce parcialmente y el $18.8 \%$ restante refirió no conocerlo. Por otra parte, el $54.3 \%$ de los universitarios, considera que existe coherencia entre el plan de estudios y el perfil de egreso de la Licenciatura, el $23.3 \%$ no sabe que tan congruente lo está, y el $22.4 \%$ respondió que no son congruentes. 
En relación a los objetivos generales, del citado Plan de estudios de las carreras respondieron de la siguiente manera: el $60.6 \%$ de los encuestados considera que los objetivos son congruentes y el $27.1 \%$ desconoce que tan congruente puedan estar éstos y $12.3 \%$ no lo conocen.

Se les preguntó a los encuestados cómo ven el mercado laboral como egresados, al respecto, el $44.2 \%$ contestaron que si hay un buen mercado laboral para su carrera, el $30.1 \%$ considera que no es tan bueno como pensaban cuando eligieron la carrera, y el $25.7 \%$ considera que los posibilidades son escasas.

\section{Profesores}

De esta valoración que tienen los alumnos sobre el interés que muestran en clases, el $48.1 \%$ de los estudiantes respondió que los profesores los toman en cuenta, el $26.1 \%$ solo parcialmente y $25.8 \%$ es muy incipiente dicha valoración. Por otra parte, el $49.8 \%$ considera que en términos generales sus profesores no atienden de manera eficiente sus dudas, el $26.2 \%$ les aclara la duda de manera parcial o solo en ocasiones y el $24 \%$ consideran que no son bien atendidos por sus profesores.

A pesar de las respuestas no tan positivas, un alto porcentaje de los alumnos (63\%) afirmaron que el profesor les da libertad para expresar opiniones, ideas y reflexiones, el $21.5 \%$ respondió que únicamente lo hacen algunos profesores y el $15.5 \%$ restante, considera que se ven limitados a este tipo de expresiones en clases.

En relación a los proyectos de investigación que realizan los profesores, el $39.6 \%$ de los alumnos mencionaron que son incluidos a los proyectos, el $32 \%$ comentó que solo en ocasiones y el $28.4 \%$ afirmó que nunca se les considera para tal actividad.

\begin{tabular}{|l|r|r|r|}
\cline { 2 - 4 } \multicolumn{1}{c|}{} & \multicolumn{1}{c|}{$\begin{array}{c}\text { Lo } \\
\text { conocen }\end{array}$} & \multicolumn{1}{c|}{$\begin{array}{c}\text { Lo conocen } \\
\text { parcialmen-te }\end{array}$} & $\begin{array}{c}\text { No lo } \\
\text { conocen }\end{array}$ \\
\hline Plan de estudios & $60.5 \%$ & $20.7 \%$ & $18.8 \%$ \\
\hline $\begin{array}{l}\text { Coherencia Plan de } \\
\text { estudios vs perfíl de } \\
\text { egreso }\end{array}$ & $54.3 \%$ & $23.3 \%$ & $22.4 \%$ \\
\hline Objetoivos generales & $60.6 \%$ & $27.1 \%$ & $12.3 \%$ \\
\hline Mercado laboral & $44.2 \%$ & $30.1 \%$ & $25.7 \%$ \\
\hline Profesores & & & \\
\hline $\begin{array}{l}\text { Valoración hacia sus } \\
\text { alumnos }\end{array}$ & $48.1 \%$ & $26.1 \%$ & $25.8 \%$ \\
\hline Dudas atendidas & $49.8 \%$ & $26.2 \%$ & $24 \%$ \\
\hline
\end{tabular}

Tabla 1 Plan de estudios y profesores

Fuente: Elaboración Propia

ISSN: 2414-8857

ECORFAN® Todos los derechos reservados

\section{Marco normativo}

Esta categoría contribuirá a valorar a partir de las opiniones de los estudiantes en qué medida tienen conocimiento de la normatividad que existe en la universidad. De manera particular en lo que se refiere a la Ley Orgánica, el 34.5\% refirió conocerla, $29.8 \%$ lo conocen parcialmente, y el $35.7 \%$ no lo conocen. Por otra parte, únicamente el $28.7 \%$ de los estudiantes refirió conocer el Estatuto General de la $\mathrm{UNACH}$, mientras que el $32.2 \%$ refiere que conoce algo, y el $39.1 \%$ restante, sabe muy poco, inclusive hay alumnos que no sabían de la existencia de dicho documento.

Respecto al conocimiento que tienen del Reglamento de evaluación profesional de los egresados, los encuestados respondieron que el $36.3 \%$ lo conocen; el $28.5 \%$ expresó conocerlo parcialmente y el $35.2 \%$ restante no lo conoce.

En cuanto al servicio social el $41.9 \%$ de los encuestados consideran conocer el reglamento; $27.7 \%$ lo conoce parcialmente y $30.4 \%$ no lo conoce.

El Reglamento académico de los alumnos, es otro de los documentos importantes en la vida académica de los alumnos; sin embargo, no todos lo conocen, ya que solo el $52.5 \%$ refirió estar seguros de conocerlo, el $24 \%$ lo conoce parcialmente, y el $23.5 \%$ a oído hablar de él pero no le ha dado la importancia que éste tiene.

Los criterios que lo estudiantes tienen para la elección de sus representantes ante el Consejo Tácnico y Universitario, el 35.9\% refiere conocerlos, el $28.7 \%$ los conoce parcialmente, el resto de los estudiante refirió no conocer los mecanismos para la elección de tan importante cargo (35.4\%), lo que debe de poner en alerta a las autoridades universitarias.

Referente al Programa institucional de tutorías; la información recabada muestra que el $34.3 \%$ conoce el programa, el $29.3 \%$ lo conoce parcialmente y el $36.4 \%$, ha oído hablar de él pero no sabe para que le va a servir. 
Para el otorgamiento de becas la Universidad cuenta con un reglamento del cual se les preguntó a los alumnos si conocen los criterios que se utilizan para la asignación de éstas; al respecto, el $51.7 \%$ de los alumnos respondieron que conocen los criterios de asignación, el 23\% lo conocen parcialmente y el $25.3 \%$ no conocen el citado reglamento.

La respuesta que se obtuvo de los encuestados marca una tendencia en cuanto al conocimiento que tienen del Reglamento interno de la biblioteca, ya que el $55.1 \%$ opinó conocer el reglamento, el $24.8 \%$ mencionó conocer parcialmente el reglamento y el $20.1 \%$ señaló no tener conocimiento; por lo que se puede deducir que únicamente una minoría no está enterado de dicho reglamento. Así mismo, se les preguntó a cerca del Reglamento interno de cómputo del cual el 50.6\% refirió conocerlo, $27.5 \%$ lo conoce parcialmente y el $21.9 \%$ dijo no conocerlo.

Por lo que corresponde al Reglamento interno de intercambio académico el $40.5 \%$ manifestaron estar informados; el $27.8 \%$ parcialmente informado y el $31.7 \%$ no lo está, o bien no tiene interés por que no desea realizar intercambio. En general, los alumnos opinaron que su Escuela o Facultad, no les ha informado a cerca de la Legislación Universitaria, ya que $48.8 \%$ así lo refirió, seguido del $28.8 \%$ que mencionó que alguna vez se los hicieron saber y únicamente el $22.4 \%$ afirmó que su Escuela o Facultad les ha informado a cerca de dicha Legislación. Al respecto, es urgente implementar un programa de difusión que garantice la comunicación de los reglamentos existentes en la Universidad.

\begin{tabular}{|l|r|r|r|}
\cline { 2 - 4 } \multicolumn{1}{l|}{} & \multicolumn{1}{c}{$\begin{array}{c}\text { Lo } \\
\text { conocen }\end{array}$} & \multicolumn{1}{c|}{$\begin{array}{c}\text { Lo conocen } \\
\text { parcialmen-te }\end{array}$} & $\begin{array}{c}\text { No lo } \\
\text { conocen }\end{array}$ \\
\hline Normatividad & & & \\
\hline Ley orgánica & $34.5 \%$ & $29.8 \%$ & $35.7 \%$ \\
\hline Estatuto General & $28.7 \%$ & $32.2 \%$ & $39.1 \%$ \\
\hline Evaluación profesional & $36.3 \%$ & $28.5 \%$ & $35.2 \%$ \\
\hline Servicio social & $41.9 \%$ & $27.7 \%$ & $30.4 \%$ \\
\hline $\begin{array}{l}\text { Reglamento académico } \\
\text { alumnos }\end{array}$ & $52.5 \%$ & $24 \%$ & $23.5 \%$ \\
\hline $\begin{array}{l}\text { Elección } \\
\text { representantes }\end{array}$ & $35.9 \%$ & $28.7 \%$ & $35.4 \%$ \\
\hline Programa de tutorias & $34.3 \%$ & $29.3 \%$ & $26.4 \%$ \\
\hline Becas a estudiantes & $51.7 \%$ & $23 \%$ & $25.3 \%$ \\
\hline $\begin{array}{l}\text { Reglamento interno de } \\
\text { la biblioteca }\end{array}$ & $55.1 \%$ & $24.8 \%$ & $20.1 \%$ \\
\hline $\begin{array}{l}\text { Reglamento interno de } \\
\text { cómputo }\end{array}$ & $50.6 \%$ & $27.5 \%$ & $21.9 \%$ \\
\hline $\begin{array}{l}\text { Reglamento interno de } \\
\text { intercambio académico }\end{array}$ & $40.5 \%$ & $27.8 \%$ & $31.7 \%$ \\
\hline $\begin{array}{l}\text { Legislación } \\
\text { Universitaria }\end{array}$ & $48.8 \%$ & $28.8 \%$ & $22.4 \%$ \\
\hline
\end{tabular}

Tabla 2 Normatividad

Fuente: Elaboración Propia

\section{Infraestructura física}

Esta categoría muestra la percepción que tienen los estudiantes respecto a las condiciones que guardan los espacios físicos de su Escuela o Facultad, así como su equipamiento, al respecto, se encontraron los siguientes resultados:

La iluminación y la áreas verdes (73\%, ambas con el mismo porcentajes), los espacios bibliotecarios (67\%), las aulas $(57.7 \%)$, los laboratorios $(57.2 \%)$ y la infraestructura tecnológica (53.7\%), los estudiante opinaron que son adecuadas. En cuanto a la cafetería desde el punto de vista de la infraestructura y no de los servicios que éstas brindan, el $69.3 \%$ considera que se encuentra en buenas condiciones.

En contra parte, los talleres, los baños, y las canchas deportivas no son los adecuados para el desarrollo integral de estudiante, con porcentajes del 58, 50.8 y 41 por ciento, en ese mismo orden para cada una de las variables; es importante aclarar que, aún cuando se les preguntó por separado el equipamiento de cada área, se percibió que la valoración de los alumnos la realizaron en su conjunto, ya que en la mayor parte de los casos preguntaron si podían opinar en general cada espacio físico.

Por último, en la mayoría de las Facultades, Escuelas y Centros, no existe un espacio exprofeso para los servicios médicos que presta la Universidad, adicionalmente, los estudiantes consideran que dichos servicios no son buenos.

\section{Agradecimiento}

Se le agradece al Programa de Mejoramiento del Profesorado (PROMEP), el apoyo financiero brindado a esta investigación.

\section{Conclusiones}

Es indispensable seguir reflexionando y teorizando en cuanto a las expectativas del estudiante universitario; lo que implica, seguir debatiendo en torno a los planteamientos que diversos autores trabajan en esta línea de investigación conceptual, con la idea de ir consolidando referentes teóricos sobre satisfacción. 
Por otra parte, es apropiado continuar trabajando acerca de las metodologías, técnicas e instrumentos para la recolección de datos que sea de utilidad no solo para continuar con esta línea temática de indagación sino que ayude en seguir construyendo metodologías apropiadas de acuerdo al contexto de cada región o institución educativa.

Con base en los resultados obtenidos con la metodología utilizada y la encuesta aplicada se puede concluir que se obtuvieron las respuestas por parte de los universitarios que participaron lo que permite hacer inferencias en relación a la percepción estudiantil que tienen y la valoración asignada a cada una de las preguntas formuladas de acuerdo a la escala de medición utilizada. Con ello se cuenta con información para estimar porcentualmente el grado de satisfacción del universitario.

Se puede presuponer que la investigación realizada y los resultados obtenidos pueden contribuir a los estudios de satisfacción de los estudiantes universitarios.

Se debe de reconocer de las limitaciones que se tuvieron para la implementación de la investigación. Se señalas algunas: el factor del tiempo y las distancias que se tienen que recorrer para la aplicación de las encuestas en las escuelas o facultades de la universidad dada la situación geográfica del estado; la autorización de las autoridades universitarias para agendar el día y hora para aplicarlas en cada escuela o facultad aunado a los trámites para tener a la mano la lista de alumnos de cada uno de los grupos seleccionados; la adecuación de la encuesta al contexto de la región y de la institución y el factor tiempo que es fundamental en este tipo de investigación.

\section{Referencias}

Alves H. y Raposo M. (2005): La Medición de la Satisfacción en la Enseñanza Universitaria: El ejemplo de la Universidade da Beira Interior.

Baeza, M. A. (2000). Los caminos invisibles de la realidad social: ensayo de sociología profunda sobre los imaginarios sociales. Ril editores.
Carretero, A. (2001). Imaginarios sociales y crítica ideológica. Una perspectiva para la comprensión de la legitimación del orden social. Santiago de Compostela: Universidad Santiago de Compostela,(Tesis doctoral inédita).

Jiménez, A., Terriquez, B. y Robles F. J. (2011). Evaluación de la satisfacción académica de los estudiantes de la Universidad Autónoma de Nayarit. Revista Fuente6, 46-56.

Jodelet, D. (1986). La representación social: fenómenos, concepto y teoría. Moscovici, Serge (comp.), Psicología Social II, Barcelona, Paidós, 469-494.

Moscovici, S. (1961). El Psicoanálisis, su Imagen y su Público, 1979 ed. Spanish. Buenos Aires: Huemul.

Surdez-Pérez, E. G., Sandoval-Caraveo, M. D. C., \& Lamoyi-Bocanegra, C. L. (2018). Satisfacción estudiantil en la valoración de la calidad educativa universitaria. Educación y Educadores, 21(1), 9-26.

Universidade da Beira Interior, Dpto. de Gestão e Economia, Covilhã (Portugal) http:// econwpa.wustl.edu:8089/eps/hew/papers/0511/ 0511004.pdf. (abril 2006).

Vergara Quintero, M. (2008). La naturaleza de las representaciones sociales. Revista Latinoamericana de Ciencias Sociales, Niñez y Juventud, 6 (1), 55-80. 